\title{
Diffusion tractography and neuromotor outcome in very preterm children with white matter abnormalities
}

\author{
Meredith E. Estep', Christopher D. Smyser ${ }^{1,2}$, Peter J. Anderson ${ }^{3,4}$, Cynthia M. Ortinau', Michael Wallendorf ${ }^{5}$, \\ Charles S. Katzman', Lex W. Doyle ${ }^{3,4,6}$, Deanne K. Thompson ${ }^{3}$, Jeffery J. Neil ${ }^{1,2,7}$, Terrie E. Inder ${ }^{1,2,7}$ and Joshua S. Shimony ${ }^{7}$
}

\begin{abstract}
BACKGROUND: Moderate-to-severe white matter abnormality (WMA) in the newborn has been shown to produce persistent disruptions in cerebral connectivity but does not universally result in neurodevelopmental disability in very preterm (VPT) children. The aims of this hypothesis-driven study were to apply diffusion imaging to: (i) examine whether bilateral WMA detected in VPT children in the newborn period can predict microstructural organization at the age of $7 \mathrm{y}$ and (ii) compare corticospinal tract and corpus callosum (CC) measures in VPT children at the age of $7 \mathrm{y}$ with neonatal WMA with normal vs. impaired motor functioning.
\end{abstract}

METHODS: Diffusion parameters of the corticospinal tract and CC were compared between VPT 7-y olds with $(n=20)$ and without $(n=42)$ bilateral WMA detected in the newborn period. For those with WMA, diffusion parameters were further examined.

RESULTS: Microstructural organization of corticospinal tract and CC tracts at the age of $7 y$ were altered in VPT children with moderate-to-severe WMA detected at term equivalent age as compared with those without injury. Furthermore, diffusion parameters differed in the CC for children with WMA categorized by motor outcome $(n=8)$.

CONCLUSION: WMA on conventional magnetic resonance imaging at term equivalent age is associated with altered microstructural organization of the corticospinal tract and CC at $7 \mathrm{y}$ of age.

$E_{t a d}^{a t}$ arly brain development can be disrupted by white matter abnormality (WMA), which is common in infants born preterm (1). Studies employing conventional (T1- and T2-weighted) magnetic resonance imaging (MRI) have demonstrated that up to $50 \%$ of very preterm (VPT) infants have WMA accompanied by neuronal or axonal disease (2). In these infants, moderate-to-severe WMAs identified on qualitative MRI assessment, including volume loss, cysts, ventriculomegaly, thinning of the corpus callosum (CC), and delayed myelination, are associated with increased risk for adverse motor outcomes in early childhood (3-6). Thus, MRI scans performed at term equivalent postmenstrual age in VPT infants may provide valuable prognostic information for clinicians $(7,8)$. However, conventional MRI studies may not fully elucidate the nature and extent of WMA.

Diffusion tensor imaging (DTI) also provides information on cerebral architecture, enabling quantification of white matter microstructural properties through the measurement of water displacement in the brain. For example, diffusivity values decrease significantly during brain maturation, reflecting changes in overall brain water content (9-11). Anisotropy measures provide information on white matter fiber bundle orientation and integrity, both of which are potentially disrupted in WMA. DTI measurements also enable calculation of quantitative diffusion tensor tractography (DTT), which has been used to detect subtle anatomic connectivity differences in white matter fiber bundles in preterm children (12). Across these measures, alterations in DTI parameters may reflect disruption in axonal and myelin microstructure (13).

It is important to note that although DTI provides information that differs from and is complementary to that obtained using conventional MRI, it is not entirely independent. The severity of white matter signal abnormalities on conventional MRI has been shown to correlate with regional DTI measures in VPT infants $(14,15)$, and changes in diffusion measures in the corticospinal tract (CST) and CC also correlate with qualitative WMA $(16,17)$. Additionally, DTT studies have revealed alterations in the development of the CST $(12,18)$ and CC $(19,20)$ in preterm adolescents and young adults with WMA.

The current study was aimed to determine whether bilateral moderate-to-severe WMA, detected on conventional MRI at term equivalent age in VPT children, can predict DTI measures at $7 \mathrm{y}$ of age in two major tracts associated with motor function, the CST and CC. Because considerable variability exists in developmental outcomes for individuals with similar qualitative MRI lesions (21-24), we then investigated whether DTI measures at the age of 7 y differed between VPT children with and without motor impairment in subjects with bilateral moderate-to-severe WMA identified at term equivalent age.

\footnotetext{
'Department of Pediatrics, Washington University, St Louis, Missouri; ${ }^{2}$ Department of Neurology, Washington University, St Louis, Missouri; ${ }^{3} \mathrm{Critical}$ Care and Neurosciences, Murdoch Children's Research Institute, Melbourne, Australia; ${ }^{4}$ Department of Paediatrics, University of Melbourne, Melbourne, Australia; ${ }^{5}$ Division of Biostatistics, Washington University, St Louis, Missouri; ${ }^{6}$ Department of Obstetrics and Gynaecology, The Royal Women's Hospital, Melbourne, Australia; ${ }^{7}$ Mallinckrodt Institute of Radiology, Washington University, St Louis, Missouri. Correspondence: Joshua S. Shimony (shimonyj@mir.wustl.edu)
} 


\section{RESULTS}

A total of sixty-two 7-y-old VPT subjects were included in the present study, including 42 subjects without WMA and 20 subjects with bilateral moderate-to-severe WMA on neonatal MRI scan. The remaining 164 subjects were ineligible for the following reasons: death $(n=2)$, incomplete data at the age of $7 \mathrm{y}(n=48)$, or mild WMA or unilateral injury $(n=114)$. There were no demographic or clinical differences between included and excluded subjects (data not shown). The median postmenstrual age at birth for included subjects was $27.3 \mathrm{wk}$ (range: 22-32 wk) and median birth weight was $937 \mathrm{~g}$ (range: $414-1,425 \mathrm{~g}$ ). Left-handedness was reported in $16.7 \%$ of the subjects without WMA and $10 \%$ of the WMA group. For the infants with bilateral moderate-to-severe WMA, four subjects had cystic periventricular leukomalacia. A total of five subjects had lesions in the area of the CST. No infants had evidence of injury in the region of the posterior limb of the internal capsule and a single subject had asymmetric myelination of the posterior limb of the internal capsule. Additional group demographic information is provided in Table 1.

At 7 y of age, VPT children with moderate-to-severe WMA on qualitative scoring of term equivalent MRI demonstrated lower fractional anisotropy (FA) and higher mean diffusivity (MD) and radial diffusivity (RD) in the CC compared with VPT children who had no WMA on neonatal MRI. The right CST also displayed higher MD and axial diffusivity (AD) in children with neonatal WMA. Notably, there were no differences between groups in the left CST (Table 2).
In relation to motor outcomes, of the $20 \mathrm{VPT}$ infants who were categorized with moderate-to-severe WMA at term equivalent age, there were 12 VPT children with no impairment on the Movement Assessment Battery for Children, 2nd Edition (MABC2) and 8 VPT children with impairment at $7 \mathrm{y}$ of age. The only value that differed between groups, when including Bonferroni correction for multiple comparisons, was lower FA in the CC in VPT children with impaired motor ability at the age of $7 \mathrm{y}$. There was no difference in the diffusion measures within the CST (Table 3).

Comparison of diffusion parameters between VPT children with and without motor impairment at $7 \mathrm{y}$ of age (irrespective of WMA status during infancy) demonstrated no differences between groups (data not shown).

In the Track-Based Spatial Statistics analysis (Figure 1), the CC demonstrated lower FA values in the moderate-to-severe WMA group ( $n=17$, after removal of 3 subjects with poor registration) as compared with subjects without WMA $(n=42)$ (areas colored yellow and red on the FA skeleton in Figure 1a). A similar pattern was identified with $\mathrm{RD}$, with greater values in the WMA group (Figure 1b). No differences in MD or AD were identified between groups. In addition, no group differences were identified for any of the CST parameters.

\section{DISCUSSION}

There are two key findings utilizing DTT in the present study. The first is that, as previously reported, VPT born children with neonatal qualitative moderate-to-severe WMAs displayed

Table 1. Group characteristics for 7-y-old VPT children with and without moderate-to-severe WMA

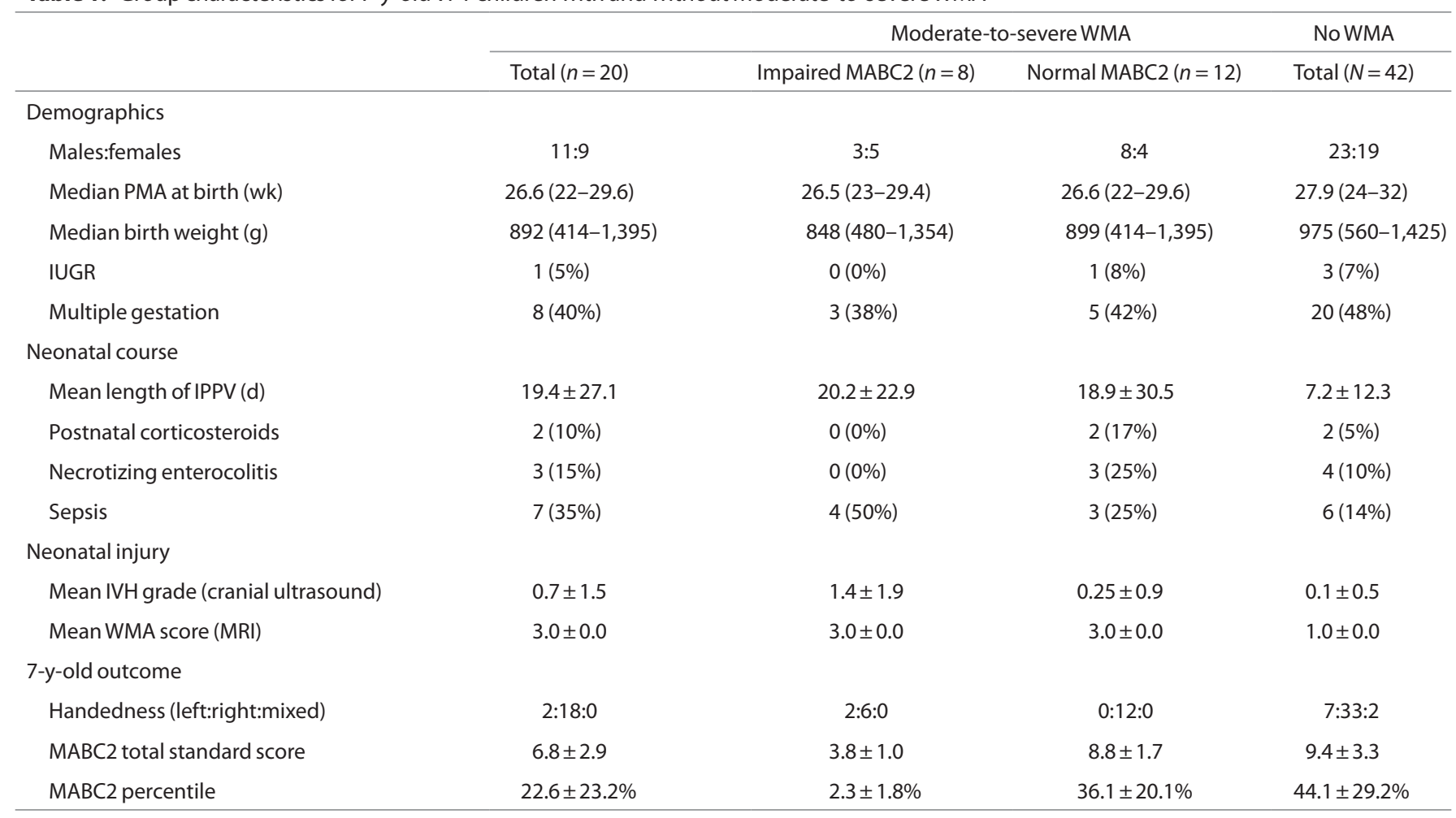

IPPV, intermittent positive pressure ventilation; IUGR, intrauterine growth restriction; IVH, intraventricular hemorrhage; MABC2, Movement Assessment Battery for Children, 2nd Edition; MRI, magnetic resonance imaging; PMA, postmenstrual age; VPT, very preterm; WMA, white matter abnormality. 
Table 2. Diffusion parameters for 7-y-old VPT children with and without moderate-to-severe WMA

\begin{tabular}{|c|c|c|c|c|}
\hline Parameter & Tract & $\begin{array}{l}\text { Moderate-to-severe } \\
\quad \text { WMA }(n=20)\end{array}$ & $\begin{array}{c}\text { No WMA } \\
(n=42)\end{array}$ & $P$ value $^{\mathrm{a}}$ \\
\hline \multirow{3}{*}{ FA } & Left CST & $0.64 \pm 0.03$ & $0.64 \pm 0.03$ & 0.95 \\
\hline & Right CST & $0.57 \pm 0.03$ & $0.58 \pm 0.03$ & 0.54 \\
\hline & CC & $0.65 \pm 0.03$ & $0.68 \pm 0.02$ & 0.0002 \\
\hline \multirow[b]{2}{*}{ MD } & Left CST & $0.76 \pm 0.02$ & $0.76 \pm 0.02$ & 0.44 \\
\hline & $\begin{array}{l}\text { Right } \\
\text { CST }\end{array}$ & $0.90 \pm 0.07$ & $0.85 \pm 0.06$ & 0.008 \\
\hline \multirow{4}{*}{$A D$} & CC & $1.07 \pm 0.08$ & $1.02 \pm 0.07$ & 0.008 \\
\hline & Left CST & $1.43 \pm 0.04$ & $1.42 \pm 0.04$ & 0.43 \\
\hline & $\begin{array}{l}\text { Right } \\
\text { CST }\end{array}$ & $1.53 \pm 0.10$ & $1.46 \pm 0.07$ & 0.009 \\
\hline & CC & $1.90 \pm 0.10$ & $1.88 \pm 0.07$ & 0.50 \\
\hline \multirow{3}{*}{$\mathrm{RD}$} & Left CST & $0.43 \pm 0.03$ & $0.43 \pm 0.03$ & 0.74 \\
\hline & Right CST & $0.59 \pm 0.07$ & $0.54 \pm 0.06$ & 0.02 \\
\hline & CC & $0.66 \pm 0.08$ & $0.59 \pm 0.07$ & 0.0007 \\
\hline
\end{tabular}

Bold text denotes significant between group differences following multiple comparisons correction with a level of significance determined at $P \leq 0.01$. FA is dimensionless. Units for MD, AD, and RD are $10^{-3} \mathrm{~mm}^{2} / \mathrm{s}$.

$A D$, axial diffusivity; $C C$, corpus callosum; $C S T$, corticospinal tract; $F A$, fractional anisotropy; $M D$, mean diffusivity; $R D$, radial diffusivity; $V P T$, very preterm; WMA, white matter abnormality.

'Adjusted for gender, gestational age at birth, and age at scan for all tracts. Additional adjustment for handedness was done only in the CST tracts.

alterations in diffusion measures within the CST and CC tracts at $7 \mathrm{y}$ of age compared with those without qualitative neonatal WMA. The second key finding, complementary to prior studies using WMA alone (8), is that $40 \%$ of VPT born children with qualitative neonatal moderate-to-severe WMA (8/20) experience significant motor impairment at $7 \mathrm{y}$ of age, and such impairment is associated with abnormal diffusion measures in the CC compared with nonimpaired children with similar patterns of qualitative neonatal bilateral white matter injury. These findings highlight that the perturbations in cerebral microstructure of the CST and CC tracts that occur secondary to neonatal moderate-to-severe WMA persist and may differentiate VPT children with motor impairments in childhood.

\section{Diffusion Measures Affected by WMA}

Corticospinal tract. This study demonstrated that VPT children with moderate-to-severe WMA demonstrated increased diffusivity measures in the right CST compared with those without, indicative of abnormal microstructural organization. This pattern of injury has been hypothesized to reflect disrupted fiber tracts, less myelin, and/or lower axonal density. This premise is consistent with animal models indicating a trophic role for oligodendrocytes in axonal development in rabbit (25) and rat embryonic cortical neurons (26) and disruption in preoligodendrocyte maturation following WMA in a neonatal rat model of hypoxic-ischemic injury (27). This pattern of injury has also been reported in a mouse model of multiple sclerosis (28).
Table 3. Diffusion parameters for 7-y-old VPT children with moderate-to-severe WMA categorized by motor outcome

\begin{tabular}{llccc}
\hline \multirow{2}{*}{ Parameter } & \multicolumn{1}{c}{ Tract } & $\begin{array}{c}\text { Impaired } \\
\text { MABC2 }(n=8)\end{array}$ & $\begin{array}{c}\text { Normal } \\
\text { MABC2 }(n=12)\end{array}$ & $P_{\text {value }}{ }^{\mathrm{a}}$ \\
\hline \multirow{2}{*}{ FA } & Left CST & $0.65 \pm 0.03$ & $0.64 \pm 0.02$ & 0.71 \\
& Right CST & $0.59 \pm 0.02$ & $0.56 \pm 0.03$ & 0.16 \\
MD & CC & $\mathbf{0 . 6 3} \pm \mathbf{0 . 0 3}$ & $\mathbf{0 . 6 6} \pm \mathbf{0 . 0 2}$ & $\mathbf{0 . 0 1}$ \\
& Left CST & $0.76 \pm 0.01$ & $0.76 \pm 0.03$ & 0.89 \\
& Right CST & $0.91 \pm 0.05$ & $0.90 \pm 0.08$ & 0.63 \\
& CC & $1.09 \pm 0.10$ & $1.06 \pm 0.06$ & 0.75 \\
AD & Left CST & $1.44 \pm 0.05$ & $1.42 \pm 0.04$ & 0.68 \\
& Right CST & $1.57 \pm 0.08$ & $1.51 \pm 0.10$ & 0.80 \\
& CC & $1.89 \pm 0.11$ & $1.91 \pm 0.09$ & 0.27 \\
& Left CST & $0.43 \pm 0.02$ & $0.43 \pm 0.03$ & 0.67 \\
& Right CST & $0.58 \pm 0.04$ & $0.59 \pm 0.08$ & 0.37 \\
& CC & $0.69 \pm 0.10$ & $0.64 \pm 0.05$ & 0.17 \\
\hline
\end{tabular}

Bold text denotes significant between group differences following multiple comparisons correction with a level of significance determined at $P \leq 0.01$. FA is dimensionless. Units for $\mathrm{MD}, \mathrm{AD}$, and $\mathrm{RD}$ are $10^{-3} \mathrm{~mm}^{2} / \mathrm{s}$.

AD, axial diffusivity; CC, corpus callosum; CST, corticospinal tract; FA, fractional anisotropy; MABC2, Movement Assessment Battery for Children, 2nd Edition; MD, mean diffusivity; RD, radial diffusivity; VPT, very preterm; WMA, white matter abnormality. adjusted for gender, gestational age at birth, and age at scan for all tracts. Additional adjustment for handedness was done only in the CST tracts.

The CST is one of the earliest areas of the brain to undergo myelination and, therefore, may be particularly vulnerable to injury. Prior investigation has shown the CST to be susceptible to the effects of WMA, with abnormal anisotropy and diffusivity measures reported in infants with periventricular leukomalacia (29) and extensive white matter signal abnormalities (14). Furthermore, it is located within the regions dorsal and lateral to the lateral ventricles, areas known to be susceptible to white matter injury. The current investigation demonstrated that these effects persist beyond infancy and into childhood (although this was noted on the right side only as discussed below).

Corpus callosum. Commissural fibers such as the CC have also been shown to be susceptible to the effects of WMA in VPT born children (30). The current study supports these findings, as the FA was reduced and diffusivity values were increased in children with moderate-to-severe WMA. These findings in the CC were also confirmed by the Track-Based Spatial Statistics analysis (Figure 1). In comparison to the corticospinal pathways, the $\mathrm{CC}$ matures relatively late, continuing to show growth secondary to myelination of interhemispheric pathways through adolescence. The susceptibility of this tract to complications associated with prematurity has been well established. Yeatman et al. (31) demonstrated alterations in callosal diffusion correlated with early WMA. In addition, Fan et al. (29) demonstrated children with periventricular leukomalacia had reduced FA in the CC. In this investigation, children with early bilateral moderate-to-severe WMA had decreased FA in the CC in comparison with children without WMA, a pattern previously reported in delayed or disrupted myelination $(32,33)$. We have previously shown that altered microstructural 


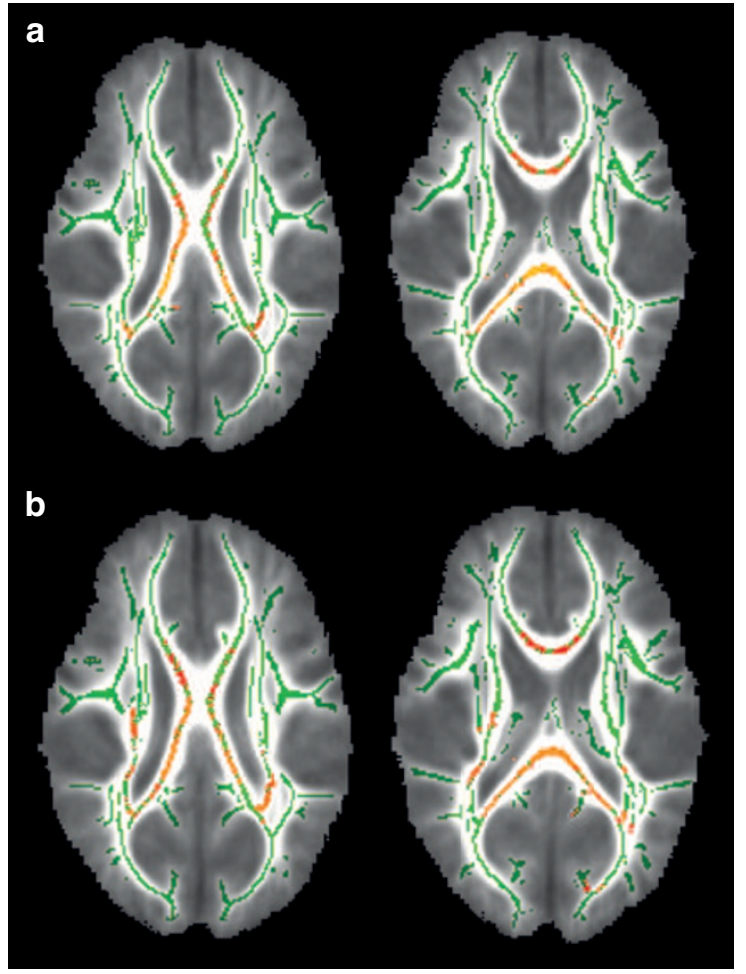

Figure 1. Track-Based Spatial Statistics (TBSS) results comparing very preterm (VPT) children with and without white matter abnormality (WMA). TBSS results denoting areas of the corpus callosum (CC) that differed significantly between VPT children scanned at $7 \mathrm{y}$ of age with and without WMA on neonatal magnetic resonance imaging. Results demonstrate (a) higher fractional anisotropy (FA) and (b) lower radial diffusivity in the CC in subjects without WMA. Yellow color denotes areas that differ between groups $(P \leq 0.05)$. Green color indicates areas with no difference. Results overlaid on averaged FA map.

organization of the CC in VPT infants is associated with WMA at term equivalent age (34), with the current study indicating this association persists into childhood.

\section{Motor Impairment}

The study demonstrated lower FA in the CC of subjects with moderate-to-severe WMA and motor impairment. These findings are consistent with studies investigating the relationship between diffusion measures of the CC and motor outcome in preterm populations. Counsell et al. and Skranes et al. reported FA within the posterior body and isthmus of the CC correlated with neurodevelopmental ability in preterm children and young adults $(32,33)$. Rose et al. (35) reported reduced FA in the splenium of the CC in VPT infants with abnormal neurodevelopment. In addition, we previously found that higher $\mathrm{MD}$ and RD within the splenium of VPT infant was related to worse motor functioning at $2 \mathrm{y}$ of age (34). These findings support that the CC plays an important function in differential motor ability in VPT born children with moderate-to-severe WMA and supports further investigative focus in this area.

\section{Hemispheric Asymmetry}

A prior study by Hasan et al. (36) demonstrated DTT asymmetry of the uncinate fasciculus across the life span, with

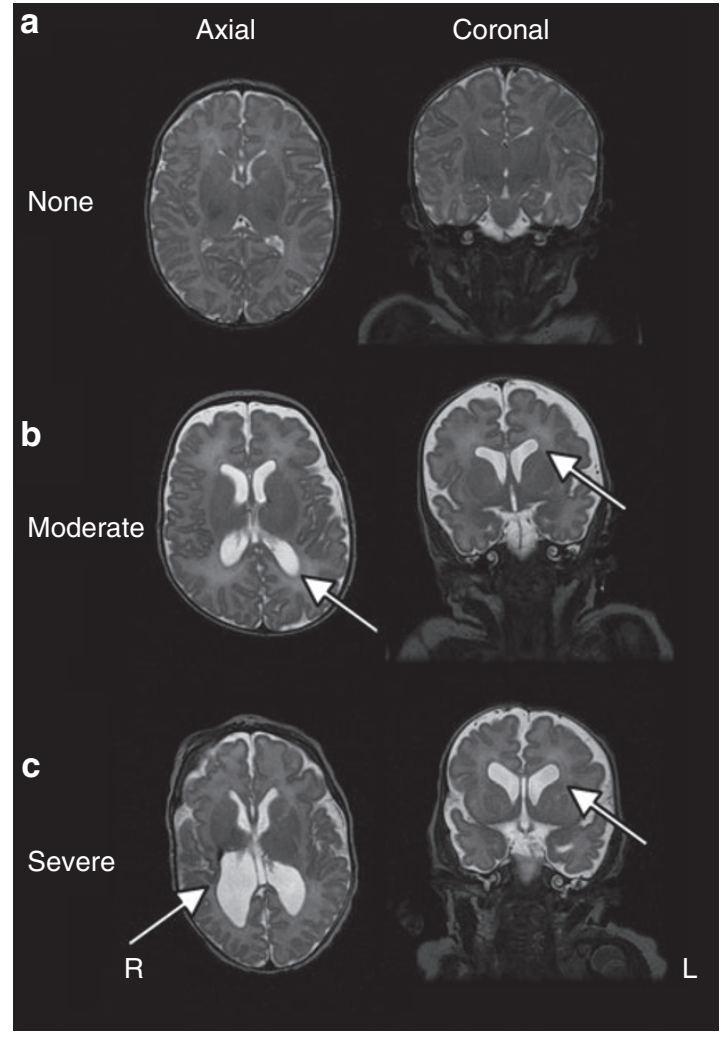

Figure 2. White matter abnormality (WMA) in preterm infants scanned at term equivalent postmenstrual age. Axial and coronal T2-weighted magnetic resonance images illustrating representative example of subjects (a) without WMA, (b) with moderate WMA, and (c) with severe WMA (arrows denote representative areas of WMA).

elevation of FA in the left CST compared with the right in a group of right-handed subjects. A study by Beaulieu et al. (37) advanced this premise by demonstrating asymmetry in the arcuate fasciculus and correlating it with cognitive language skills in typically developing children. Both studies imply that diffusion asymmetry is related to remodeling due to asymmetric activity in right-handed subjects.

The findings in the CST of neonates with WMA were confined to the right (nondominant) side of the brain. This potentially reflects asymmetric recovery due to differences in activity on the dominant side of the brain. It is also plausible that functional adaptation for motor performance is dependent on adequate compensation involving the nondominant hemisphere. Subsequently, if this area is also affected, the potential for positive adaptation is limited. The current investigation was unable to test this hypothesis in the neonatal period due to limitations in the DTI acquisition, but it would be worthy of further study in preterm infants at term in relation to motor development.

\section{Strengths and Limitations}

The present study benefits from its longitudinal design, enabling exploration of the relationship between MRI results from the neonatal period with neuroimaging and neurodevelopmental outcome measures at $7 \mathrm{y}$ of age. However, the study focused on a specific subset of subjects from a larger cohort, 


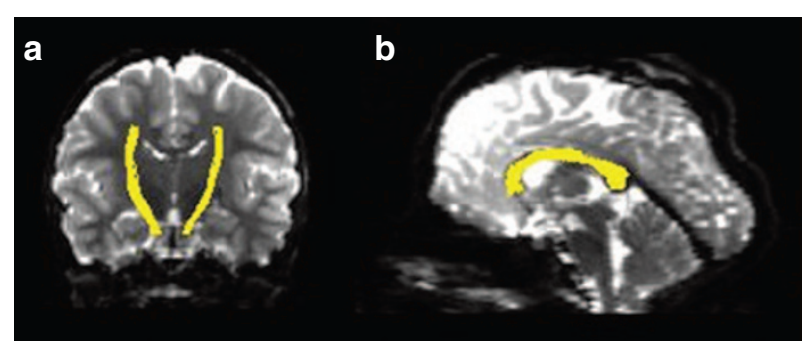

Figure 3. Region of interest-based tractography for very preterm children scanned at 7 y of age. Images illustrating diffusion tensor tractography results for the (a) corticospinal tract and (b) corpus callosum for a 7-y-old subject without white matter abnormality. Results of tracks are overlaid in yellow on diffusion weighted image.

which limited subject numbers available for subgroup analyses. Further studies of children with unilateral injury and those with less severe WMA are needed. In addition, subjects were studied during a period in which heterogeneity may exist due to differing rates of individual development, introducing an additional confound into the interpretation of the results. Further follow-up of these and other cohorts during later developmental periods is warranted.

There are also technical limitations inherent to DTT that should be noted. When applying this technique, there is no measure indicative of the confidence in the reconstructed diffusion trajectories. Nonetheless, for regions where there is a single dominant fiber pathway (such as the CST and CC), uncertainty in the principal diffusion direction is small.

\section{Conclusion}

WMA on qualitative conventional MRI at term equivalent age is correlated with persistent microstructural defects at $7 \mathrm{y}$ of age and associated with impaired motor outcome. This implies that white matter pathways play a critical role in the pathophysiology of motor disability. The study results also suggest developmental adaptation to injury, with microstructural differences identified in subjects with normal outcomes despite significant neonatal WMA. This highlights the plasticity inherent in this population and represents a possible marker for future therapeutic interventions.

\section{METHODS}

\section{Participants}

Two hundred and twenty-seven VPT subjects (born $<32 \mathrm{wk}$ of gestational age or having $<1,250 \mathrm{~g}$ birth weight) with no genetic or congenital abnormalities were recruited for the Victorian Infant Brain Study (VIBeS), a prospective, longitudinal cohort study conducted between 2001 and 2003 at the Royal Women's Hospital, Victoria, Australia. For this investigation, all subjects with either bilateral qualitative moderate-to-severe WMA or with no WMA on neonatal MRI were eligible.

Parental informed consent was obtained for each subject prior to participation in the study. The study was approved by the Royal Women's Hospital and Royal Children's Hospital Human Research Ethics Committees.

\section{Neonatal MRI}

For all participants, brain MRI was performed at term equivalent age (37-42 wk of postmenstrual age) using a $1.5 \mathrm{~T}$ General Electric Signa LX Echospeed System MR scanner (Milwaukee, $\mathrm{WI}$ ). Acquired sequences included 3D T1 spoiled gradient recalled images $\left(0.8-1.6 \mathrm{~mm}\right.$ coronal slices, flip angle: $45^{\circ}$, retention time: $35 \mathrm{~ms}$, echo time: $9 \mathrm{~ms}$, field of view: $210 \times 157 \mathrm{~mm}^{2}$; matrix: $256 \times 192)$ and T2 dual echo (interleaved acquisition) fast recovery fast spin-echo images $(1.7-3.0 \mathrm{~mm}$ coronal slices, retention time: $4,000 \mathrm{~ms}$, echo time: $60 / 160 \mathrm{~ms}$, field of view: $220 \times 165 \mathrm{~mm}^{2}$, matrix: $256 \times 192$ interpolated to $512 \times 512)$. Scanning was performed without sedation (38).

T1- and T2-weighted images were assessed for WMA according to a standardized scoring system previously validated in this population $(3,8)$. Severity of WMA was categorized according to total WMA score on a scale of 1-4 (1: none, 2: mild, 3: moderate, 4: severe) based upon variables including presence and severity of white matter signal abnormality, white matter volume reduction, cystic white matter injury, ventriculomegaly, myelination of the posterior limb of the internal capsule, and thinning of the CC. All scans were scored by a pediatric neurologist and a neonatologist blinded to clinical status. Interrater agreement was $96 \%$ (8). Figure 2 provides representative examples of subjects with moderate-to-severe WMA.

\section{7-y-Old MRI}

At $7 \mathrm{y}$ of age, all participants underwent repeat neuroimaging using a 3T Siemens scanner at the Royal Children's Hospital, Melbourne. Data acquisition included sagittal 3D turbo spin-echo T2-weighted images (T2W) (0.9- $\mathrm{mm}^{3}$ isotropic voxels, retention time: $3,200 \mathrm{~ms}$, echo time: $447 \mathrm{~ms}$, field of view: $240 \times 215 \mathrm{~mm}^{2}$, matrix: $256 \times 230$ ), and a spin-echo echo-planar sequence with 25 noncollinear diffusion gradient directions $\left(1.7-\mathrm{mm}^{3}\right.$ isotropic voxels, retention time: $12,000 \mathrm{~ms}$, echo time: $96 \mathrm{~ms}$, field of view: $250 \times 250 \mathrm{~mm}^{2}$, matrix: $144 \times 144)$. The $b$ values for these 25 directions were chosen to linearly range from 0 to $1,200 \mathrm{~s} / \mathrm{mm}^{2}$. This choice of $b$ values differs from that used in most DTI experiments in which one or more $b=0$ values are used and a constant high $b$ value is used for all remaining directions. This choice is helpful in calculating more complicated and nonlinear diffusion tensor models.

\section{DTT Analysis}

DTI and DTT computations followed the methods detailed in Shimony et al. (39). The unsensitized $\left(b=0 \mathrm{~s} / \mathrm{mm}^{2}\right)$ DTI volume was registered to the $\mathrm{T} 2 \mathrm{~W}$ image with 12-parameter affine transform to compensate for echo planar imaging distortion. Internal head motion correction of the DTI data was performed using 9-parameter affine transform among groups of images with similar sensitization. The tensor was calculated using log-linear regression (40). Measured diffusion parameters included FA, MD, AD, and RD. Deterministic tracking algorithms and tract reconstructions were based on the streamline algorithm, i.e., by propagating along the local diffusion tensor principal eigenvector (39). The propagation increment was $0.5 \mathrm{~mm}$, and the track termination criteria include FA $<0.13$ and a radius of curvature $<1 \mathrm{~mm}$. Full brain DTT on a $1 \mathrm{~mm}^{3}$ grid was filtered by regions of interest (ROIs) manually placed perpendicular to the length of the tract based on anatomical landmarks in native space. Using a multi-ROI approach (41), the CST was identified using ROIs in the centrum semiovale and the level of the ventral pontine crossing fibers (representative example provided in Figure 3a).

The medial portion of the full CC was tracked by placing a single ROI on a midline sagittal slice and ROIs on the sagittal view that were displaced $1 \mathrm{~cm}$ to the left and to the right from the midline slice. To ensure acceptable ROI placement, the callosal radiations were visualized in each subject. The filtered tracts were then clipped outside of the limiting ROIs and designated tracking thresholds were implemented. This approach identified the central fibers of each reconstructed tract (representative example provided in Figure 3b). Diffusion parameters were averaged over the reconstructed tract area, with $\mathrm{FA}, \mathrm{MD}, \mathrm{AD}$, and $\mathrm{RD}$ measured along each tract.

In order to confirm our DTT findings, whole brain analysis of the DTI data was also performed using the method of Track-Based Spatial Statistics (42). Using each individual's motion-corrected, aligned, and averaged DTI data set, FA maps were used to create a target template for registration. FA images in the target group were aligned to each other and to a target image. The target image was the image that required the least amount of warping for all other FA 
images to align to it. The target image was affine-aligned into MNI152 standard space, and every FA image was then transformed into $1 \times 1$ $\times 1 \mathrm{~mm}^{3}$ MNI152 space. A mean FA image was calculated and used to produce the mean FA skeleton to represent the center of white matter tracts. FA images were projected onto the mean FA skeleton and thresholded at FA $>0.20$ for voxelwise analyses. The registrations for all subjects were visually inspected for accuracy, and three of the subjects from the moderate-to-severe WMA group were removed due to poor registration. $\mathrm{FA}, \mathrm{MD}, \mathrm{AD}$, and $\mathrm{RD}$ images were also calculated and all were analyzed with Randomise, a permutation-based multiple comparisons corrected statistical approach, to evaluate for differences between subjects with moderate-to-severe WMA and those without WMA.

\section{7-y-Old Developmental Testing}

At $7 \mathrm{y}$ of age, motor functioning was assessed with the MABC2 (43). This tool provides a standardized assessment of both gross and fine motor development. Children were classified as having impaired motor performance if their total motor score was at or below the 15th percentile based on the test norms. The hemisphere contralateral to the hand the child used to write during the 7-y follow-up assessment was designated as dominant.

\section{Statistical Analyses}

Linear mixed models with subject ID nested within white matter injury (or motor impairment) as a random effect were used to assess the differences in diffusion parameters across groups. Study subject ID was also included as a random variable to account for the repeated measure design. Additional multivariable analyses controlling for gender, postmenstrual age at birth, age at scan, and handedness in the CST were conducted. Due to the number of diffusion measures that were simultaneously analyzed in the four regions, Bonferroni multiple comparisons correction was applied with a level of significance determined at $P \leq 0.01$.

\section{STATEMENT OF FINANCIAL SUPPORT}

The study was funded by the National Institutes of Health (Bethesda, MD) (R01 HD05709801, P30 HD062171, K23 HD053212, K12 NS001690, and UL1 TR000448), Doris Duke Foundation Distinguished Clinical Scientist Award (New York, NY), National Medical and Health Research Council of Australia (Canberra, Australia) (Project Grant: 491209, Senior Research Fellowship: 628371), and the Victorian Government's Operational Infrastructure Program (Melbourne, Australia). This project was supported by the Intellectual and Developmental Disabilities Research Center at Washington University (NIH/NICHD P30 HD062171).

\section{ACKNOWLEDGMENTS}

We thank the subjects and their families for participation, Merilyn Bear for assisting in recruiting and retaining the participating families, and Tara Smyser for assistance with data analysis.

Disclosure: The authors declare no conflict of interest.

\section{REFERENCES}

1. Rees $\mathrm{S}$, Inder T. Fetal and neonatal origins of altered brain development. Early Hum Dev 2005;81:753-61.

2. Volpe JJ. Neurology of the Newborn. Philadelphia, PA: Elsevier, 2008.

3. Inder TE, Wells SJ, Mogridge NB, Spencer C, Volpe JJ. Defining the nature of the cerebral abnormalities in the premature infant: a qualitative magnetic resonance imaging study. J Pediatr 2003;143:171-9.

4. Krishnan ML, Dyet LE, Boardman JP, et al. Relationship between white matter apparent diffusion coefficients in preterm infants at term-equivalent age and developmental outcome at 2 years. Pediatrics 2007;120: e604-9.

5. Miller SP, Ferriero DM, Leonard C, et al. Early brain injury in premature newborns detected with magnetic resonance imaging is associated with adverse early neurodevelopmental outcome. J Pediatr 2005;147:609-16.

6. Spittle AJ, Cheong J, Doyle LW, et al. Neonatal white matter abnormality predicts childhood motor impairment in very preterm children. Dev Med Child Neurol 2011;53:1000-6.
7. van Kooij BJ, van Handel M, Nievelstein RA, Groenendaal F, Jongmans MJ, de Vries LS. Serial MRI and neurodevelopmental outcome in 9- to 10-yearold children with neonatal encephalopathy. J Pediatr 2010;157:221-227.e2.

8. Woodward LJ, Anderson PJ, Austin NC, Howard K, Inder TE. Neonatal MRI to predict neurodevelopmental outcomes in preterm infants. N Engl J Med 2006;355:685-94.

9. Hüppi PS, Maier SE, Peled S, et al. Microstructural development of human newborn cerebral white matter assessed in vivo by diffusion tensor magnetic resonance imaging. Pediatr Res 1998;44:584-90.

10. McKinstry RC, Mathur A, Miller JH, et al. Radial organization of developing preterm human cerebral cortex revealed by non-invasive water diffusion anisotropy MRI. Cereb Cortex 2002;12:1237-43.

11. McKinstry RC, Miller JH, Snyder AZ, et al. A prospective, longitudinal diffusion tensor imaging study of brain injury in newborns. Neurology 2002;59:824-33.

12. Trivedi R, Agarwal S, Shah V, et al. Correlation of quantitative sensorimotor tractography with clinical grade of cerebral palsy. Neuroradiology 2010;52:759-65.

13. Vangberg TR, Skranes J, Dale AM, Martinussen M, Brubakk AM, Haraldseth O. Changes in white matter diffusion anisotropy in adolescents born prematurely. Neuroimage 2006;32:1538-48.

14. Cheong JL, Thompson DK, Wang HX, et al. Abnormal white matter signal on MR imaging is related to abnormal tissue microstructure. AJNR Am J Neuroradiol 2009;30:623-8.

15. Hüppi PS, Murphy B, Maier SE, et al. Microstructural brain development after perinatal cerebral white matter injury assessed by diffusion tensor magnetic resonance imaging. Pediatrics 2001;107:455-60.

16. Gupta RK, Saksena S, Hasan KM, et al. Focal Wallerian degeneration of the corpus callosum in large middle cerebral artery stroke: serial diffusion tensor imaging. J Magn Reson Imaging 2006;24:549-55.

17. Møller M, Frandsen J, Andersen G, Gjedde A, Vestergaard-Poulsen P, Østergaard L. Dynamic changes in corticospinal tracts after stroke detected by fibretracking. J Neurol Neurosurg Psychiatry 2007;78:587-92.

18. Thomas B, Eyssen M, Peeters R, et al. Quantitative diffusion tensor imaging in cerebral palsy due to periventricular white matter injury. Brain 2005;128(Pt 11):2562-77.

19. Kontis D, Catani M, Cuddy M, et al. Diffusion tensor MRI of the corpus callosum and cognitive function in adults born preterm. Neuroreport 2009;20:424-8.

20. Mullen KM, Vohr BR, Katz KH, et al. Preterm birth results in alterations in neural connectivity at age 16 years. Neuroimage 2011;54:2563-70.

21. Ashwal S, Russman BS, Blasco PA, et al.; Quality Standards Subcommittee of the American Academy of Neurology; Practice Committee of the Child Neurology Society. Practice parameter: diagnostic assessment of the child with cerebral palsy: report of the Quality Standards Subcommittee of the American Academy of Neurology and the Practice Committee of the Child Neurology Society. Neurology 2004;62:851-63.

22. Dyet LE, Kennea N, Counsell SJ, et al. Natural history of brain lesions in extremely preterm infants studied with serial magnetic resonance imaging from birth and neurodevelopmental assessment. Pediatrics 2006;118:53648.

23. Ment LR, Vohr B, Allan W, et al. Change in cognitive function over time in very low-birth-weight infants. JAMA 2003;289:705-11.

24. Robinson MN, Peake LJ, Ditchfield MR, Reid SM, Lanigan A, Reddihough DS. Magnetic resonance imaging findings in a population-based cohort of children with cerebral palsy. Dev Med Child Neurol 2009;51:3945.

25. Drobyshevsky A, Song SK, Gamkrelidze G, et al. Developmental changes in diffusion anisotropy coincide with immature oligodendrocyte progression and maturation of compound action potential. J Neurosci 2005;25:598897.

26. Wilkins A, Majed H, Layfield R, Compston A, Chandran S. Oligodendrocytes promote neuronal survival and axonal length by distinct intracellular mechanisms: a novel role for oligodendrocyte-derived glial cell linederived neurotrophic factor. J Neurosci 2003;23:4967-74.

27. Back SA, Han BH, Luo NL, et al. Selective vulnerability of late oligodendrocyte progenitors to hypoxia-ischemia. J Neurosci 2002;22:455-63. 
28. Song SK, Sun SW, Ramsbottom MJ, Chang C, Russell J, Cross AH. Dysmyelination revealed through MRI as increased radial (but unchanged axial) diffusion of water. Neuroimage 2002;17:1429-36.

29. Fan GG, Yu B, Quan SM, Sun BH, Guo QY. Potential of diffusion tensor MRI in the assessment of periventricular leukomalacia. Clin Radiol 2006;61: 358-64.

30. Nagae LM, Hoon AH Jr, Stashinko E, et al. Diffusion tensor imaging in children with periventricular leukomalacia: variability of injuries to white matter tracts. AJNR Am J Neuroradiol 2007;28:1213-22.

31. Yeatman JD, Ben-Shachar M, Bammer R, Feldman HM. Using diffusion tensor imaging and fiber tracking to characterize diffuse perinatal white matter injury: a case report. J Child Neurol 2009;24:795800 .

32. Counsell SJ, Edwards AD, Chew AT, et al. Specific relations between neurodevelopmental abilities and white matter microstructure in children born preterm. Brain 2008;131(Pt 12):3201-8.

33. Skranes J, Vangberg TR, Kulseng S, et al. Clinical findings and white matter abnormalities seen on diffusion tensor imaging in adolescents with very low birth weight. Brain 2007;130(Pt 3):654-66.

34. Thompson DK, Inder TE, Faggian N, et al. Corpus callosum alterations in very preterm infants: perinatal correlates and 2 year neurodevelopmental outcomes. Neuroimage 2012;59:3571-81.

35. Rose J, Butler EE, Lamont LE, Barnes PD, Atlas SW, Stevenson DK. Neonatal brain structure on MRI and diffusion tensor imaging, sex, and neurodevelopment in very-low-birthweight preterm children. Dev Med Child Neurol 2009;51:526-35.

36. Hasan KM, Iftikhar A, Kamali A, et al. Development and aging of the healthy human brain uncinate fasciculus across the lifespan using diffusion tensor tractography. Brain Res 2009;1276:67-76.

37. Beaulieu C, Plewes C, Paulson LA, et al. Imaging brain connectivity in children with diverse reading ability. Neuroimage 2005;25:1266-71.

38. Mathur AM, Neil JJ, McKinstry RC, Inder TE. Transport, monitoring, and successful brain MR imaging in unsedated neonates. Pediatr Radiol 2008;38:260-4.

39. Shimony JS, Burton H, Epstein AA, McLaren DG, Sun SW, Snyder AZ. Diffusion tensor imaging reveals white matter reorganization in early blind humans. Cereb Cortex 2006;16:1653-61.

40. Basser PJ, Mattiello J, LeBihan D. Estimation of the effective self-diffusion tensor from the NMR spin echo. J Magn Reson B 1994;103:247-54.

41. Conturo TE, Lori NF, Cull TS, et al. Tracking neuronal fiber pathways in the living human brain. Proc Natl Acad Sci U S A 1999;96:10422-7.

42. Smith SM, Jenkinson M, Johansen-Berg H, et al. Tract-based spatial statistics: voxelwise analysis of multi-subject diffusion data. Neuroimage 2006;31:1487-505.

43. Schoemaker MM, Niemeijer AS, Flapper BC, Smits-Engelsman BC. Validity and reliability of the Movement Assessment Battery for Children-2 Checklist for children with and without motor impairments. Dev Med Child Neurol 2012;54:368-75. 